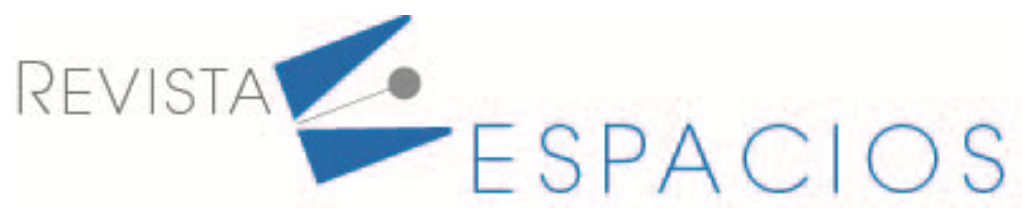

\title{
Satisfacción de los usuarios y la calidad de atención que se brinda en la Unidad de Salud Cuba Libre 24 horas
}

\section{User satisfaction and the quality of care provided at the Cuba Libre 24-hour Health Unit}

MERO, Lisseth $\mathrm{C}^{1}$

ZAMBRANO, María I. ${ }^{2}$

BRAVO, María A. ${ }^{3}$

\begin{abstract}
Resumen
Los servicios que presta la unidad médica Cuba Libre 24 horas, genera inconformidades ciudadanas. El objetivo es evaluar la percepción de calidad en la atención que se brinda. El enfoque metodológico aplicado fue cuantitativo transversal-descriptivo, utilizando la herramienta de la encuesta para el cálculo del índice de percepción de satisfacción del usuario externo, se evaluó en términos generales a la organización, cuyos resultados indican que los servicios que se relacionan directamente con el usuario de salud son negativos.

Palabras clave: servicios, usuario, calidad

Abstract

The services provided by the Cuba Libre 24-hour medical unit generate public disagreements. The objective is to evaluate the perception of quality in the care provided. The methodological approach applied was quantitative, cross-descriptive, using the survey tool to calculate the external user satisfaction perception index, the organization was evaluated in general terms, the results of which indicate that the services that are directly related to the user health are negative.

Key words: services, user, quality
\end{abstract}

\section{Introducción}

La satisfacción del usuario es uno de los aspectos que, en términos de evaluación de los servicios de salud y calidad de atención, ha venido cobrando mayor atención en salud pública, siendo considerada desde hace poco más de una década uno de los ejes de evaluación de servicios de salud (Seclén-Palacin \& Darras, 2005). En distintas organizaciones públicas y privadas emplean un sistema o herramienta para medir el indicador de satisfacción del cliente en cuanto al servicio brindado, a fin de conocer cuáles son aquellas situaciones en donde el usuario externo se siente inconforme o insatisfecho.

\footnotetext{
${ }^{1}$ Estudiante de la Maestría en Gestión del Talento Humano, Instituto de Posgrado, Universidad Técnica de Manabí. https://orcid.org/0000-0001-55514568. liscarol-1@hotmail.com

2 Docente investigador. Carrera Administración de empresas. Universidad Técnica de Manabí. https://orcid.ort/0000-0003-4407-1460. ines.zambrano@utm.edu.ec

${ }^{3}$ Docente investigador. Carrera Administración de empresas. Universidad Técnica de Manabí. https://orcid.org/0000-0002-6245-3190. mabravo@utm.edu.ec
} 
En muchas organizaciones se busca emplear la calidad en todos los procesos, el Centro de Salud Cuba Libre 24 horas, no está exento de este tema, es complicado implementar un sistema de control de calidad en todas las áreas, pues, se requiere de mucha disciplina, vigilancia, supervisiones constantes.

La calidad es un sistema de pensamiento donde la excelencia rige las decisiones y actividades de todos y cada uno de los integrantes de la organización y en todos los niveles, incluidas la dirección que debe formular políticas de calidad y constituirse en impulsadora del cambio y garante del sistema de seguridad (Varo, 1994).

La calidad de la atención en salud es un tema que adquiere cada vez más relevancia debido a la libertad con que opinan los usuarios acerca de los diferentes servicios recibidos por las instituciones proveedoras de los mismos. Esta situación está condicionada por los retos organizacionales y la creciente presión social determinada por la toma de conciencia de una sociedad más informada acerca de sus derechos (Guzmán, Ramos-Córdova, Castañeda-Sánchez, del Castillo-Sánchez, \& Gómez-Alcalá, 2006).

La calidad se puede entender como el juicio de un consumidor acerca de la excelencia total o superioridad de un producto o servicio citado por (Fariño Cortez, Cercado Mancero, Vera Lorenti, Valle Flores, \& Ocaña Ocaña, 2018). La calidad dentro del servicio de la salud es uno de los temas más importantes a tratar, considerando que el usuario es nuestro cliente y debe ser atendido en forma oportuna y con calidad.

Para la Organización Mundial de la Salud (OMS), la calidad requerida en los centros de servicios a la salud humana, sean éstas públicas o privadas deben asegurar "que el paciente logre recibir de manera óptima los servicios de valoración, diagnóstico y procesos terapéuticos más adecuados, para lograr una atención de calidad". La calidad recae en las percepciones de las personas que son atendidas, es decir en todas las acciones que se realizan, orientadas a mejorar la calidad (Donabedian, 1993). De esta manera se organizan personas y recursos para obtener los mejores resultados en términos de efectividad, eficiencia, satisfacción de profesionales, usuarios y de impacto social en la que para la calidad en salud es "la atención médica que se da como el tratamiento que proporciona un profesional de la salud a un episodio de enfermedad claramente establecido, en un paciente dado, del cual se originan dos aspectos: el primero, como la atención técnica, que es la aplicación de la ciencia y tecnología para la resolución de un problema de salud, y el segundo, como la relación interpersonal, que es la interacción social, cultural y económica entre el profesional de la salud y el paciente" (Fariño Cortez et al., 2018).

La Dirección Nacional de Calidad de los Servicios de Salud tiene dentro de sus objetivos, garantizar la calidad, así como la eficiencia y efectividad de los procesos inherentes a la prestación de los servicios de salud por parte del Ministerio de Salud Pública (MSP). Por consiguiente, dentro del marco de los derechos amparados en la Constitución del 2008, es preciso lograr la excelencia en los servicios; conforme a las políticas sectoriales, modelos de calidad, normativas y lineamientos estratégicos establecidos.

Dentro del Plan Nacional del Buen Vivir (PNBV) del Ecuador (Vivir, 2013), se ha considerado como tema principal el reposicionamiento del sector social y la salud como eje fundamental del desarrollo y bienestar de los habitantes de manera general. Actualmente, el Gobierno Nacional del Ecuador y el Ministerio de Salud Pública, como autoridad sanitaria, enfatizan en el cuidado de la salud como eje estratégico del desarrollo del país dado que los lineamientos normativos son más claros y coherentes, haciendo que se facilite la implementación de los diversos estándares de calidad en la atención.

La unidad operativa Cuba Libre 24 horas, se encuentra ubicado en el cantón Manta, parroquia Eloy Alfaro, tiene una población asignada de acuerdo a la distribución del Instituto Nacional de Estadísticas y Censo, de 8000 habitantes. Cabe recalcar que la realidad es totalmente distinta, debido a que abarca con poblaciones aledañas y de otras comunidades por ser un centro médico público de 24 horas de atención. 
La gestión debe adaptarse a las nuevas demandas y exigencias determinadas por los cambios demográficos y epidemiológicos, la prevalencia de enfermedades crónicas, la persistencia de enfermedades infecciosas y prevenibles que hacen presión sobre los servicios de salud (Arbeláez-Rodríguez \& Mendoza, 2017).

\section{Metodología}

La investigación se desarrolla con la metodología transversal-descriptiva enfoque cuantitativo, la construcción del índice de percepción al usuario/a externo se lo realiza mediante el uso del Índice de Promotores Netos o Índice Neto Promotor u originalmente conocido como Net Promoter Score (NPS) desarrollado por Frederik Reichheld. Para la metodología se consideran las preguntas de tipo escala que se encuentran dentro de la encuesta de satisfacción (Pública, 2019)

- $\quad$ Tiempo de espera-cita/turno1

- $\quad$ Tiempo de espera en el establecimiento

- $\quad$ Facilidad de llegar al establecimiento

- $\quad$ Entendimiento de la explicación del profesional

- $\quad$ Trato recibido por parte del personal

- $\quad$ Aseo y presentación del personal

- $\quad$ Limpieza del establecimiento

- $\quad$ Presentación de las instalaciones

- $\quad$ Estado del mobiliario

- Señaléticas existentes

- $\quad$ Servicios recibidos.

Desde el año 2015 las unidades de salud ejecutan encuestas como instrumento de recolección de datos con la finalidad de medir la satisfacción del usuario, ya que es un indicador importante para conocer la opinión del usuario del servicio recibido, desde sus expectativas y percepciones, dicha encuesta permite medir las dimensiones de la calidad, efectividad, eficiencia, accesibilidad, aceptabilidad, equidad, seguridad, continuidad, y oportunidad, que engloban la satisfacción del usuario como aporte a la gestión de procesos de calidad, se utiliza muestra de 318 usuarios.

\subsection{Cálculo del índice neto promotor}

\section{Cálculo del Net Promoter Score (NPS) o Índice Neto Promotor (INP)}

Para la aplicación del Índice Neto Promotor-INP es necesario contar con una matriz cuadrada Xn_m, donde:

$\mathrm{n}=$ indica el número de encuestados (filas)

$\mathrm{m}$ = el número de variables, 11 o 12 dependiendo si es hospitalización/emergencia o consulta externa. Dependiendo de la respuesta dada por los usuarios estos se clasifican en una escala dividida en 3 secciones:

\section{- $\quad$ Detractores \\ - Indiferentes \\ - $\quad$ Promotores}

Detractores: Los que califican de 0-6 (no satisfactorio), consideran que el servicio brindado no es satisfactorio o están descontentos con algún aspecto de la organización, servicio o marca, son ellos quienes pueden llegar a mejorar o a desacreditar a la institución, por la prestación de sus servicios.

Indiferentes: Los encuestados que otorgan una puntuación de 7 y 8 se conocen como Pasivos. Ellos no están incluidos en la fórmula para el cálculo de Net Promoter Score, pues se consideran "neutros" o virtualmente 
indecisos aunque pueden ser considerados de manera cualitativa fuera de la fórmula como posibles prospectos o público al que se puede convencer para convertirlos en promotores.

Promotores: Los que califican de 9-10, son las personas que se encuentran completamente satisfechos con la institución y proporcionan el mayor beneficio para el negocio, pueden llegar a promover y recomendar la compañía con otras personas, convirtiéndose así en un activo de valor para cualquier negocio.

Fórmulas

Para el cálculo se utilizan las siguientes formulas:

Detractores $=? ? 1 \leq 6+? ? 2 \leq 6+? ? 3 \leq 6+\cdots \ldots ? ? 12 \leq 6$

Indiferentes $=(? ? 1=7+? ? 2=7+? ? 3=7+\cdots \ldots ? ? 12=7)+(? ? 1=8+? ? 2=8+? ? 3=8+\cdots \ldots$ ??12 = 8) Total \# variables $=? ? 1+? ? 2+? ? 3+\cdots \ldots ? ? 12$

Promotores $=? ? 1 \geq 9+? ? 2 \geq 9+? ? 3 \geq 9+\cdots \ldots ? ? 12 \geq 9$

Para el porcentaje de cada uno de estos realizamos lo siguiente:

$\%$ Detractores. $=$ Detractores $/$ Total de $\#$ de variables $* 100$

$\%$ Indiferentes. $=$ Indiferentes $/$ Total de \# de variables *100

$\%$ Promotores. $=$ Promotores $/$ Total de \# de variables $* 100$

\section{Resultados}

La puntuación se calcula tomando el porcentaje de promotores y se le resta el porcentaje de detractores, la puntuación está entre -100 y 100.

Índice de percepción = \%Promotores - Detractores\%

Tabla 1

Índice de percepción área de consulta externa

\begin{tabular}{|c|c|c|c|c|c|c|c|}
\hline Preguntas & $\begin{array}{c}\text { Detractores } \\
0-6\end{array}$ & $\begin{array}{c}\text { Indiferentes } \\
7-8\end{array}$ & $\begin{array}{c}\text { Promotores } \\
9-10\end{array}$ & $\begin{array}{c}\% \\
\text { Detractores }\end{array}$ & $\begin{array}{c}\% \\
\text { Indiferentes }\end{array}$ & $\begin{array}{c}\% \\
\text { Promotores }\end{array}$ & $\begin{array}{c}\text { Índice De } \\
\text { Percepción }\end{array}$ \\
\hline $\begin{array}{l}\text { 6.- satisfecho } \\
\text { tiempo espera de } \\
\text { cita }\end{array}$ & 122 & 88 & 65 & 44 & 32 & 24 & -20 \\
\hline $\begin{array}{l}\text { 7.- satisfecho } \\
\text { tiempo espera } \\
\text { establecimiento }\end{array}$ & 80 & 119 & 76 & 29 & 43 & 28 & -1 \\
\hline $\begin{array}{l}\text { 8.- facilidad Ilegar } \\
\text { al } \\
\text { establecimiento }\end{array}$ & 37 & 122 & 116 & 13 & 44 & 42 & 29 \\
\hline $\begin{array}{l}\text { 12.- explicación } \\
\text { estado de salud }\end{array}$ & 6 & 87 & 182 & 2 & 32 & 66 & 64 \\
\hline 13.- trato & 3 & 88 & 184 & 1 & 32 & 67 & 66 \\
\hline $\begin{array}{l}\text { 16.- aseo } \\
\text { presentación } \\
\text { personal } \\
\text { funcionario } \\
\text { atención }\end{array}$ & 8 & 109 & 158 & 3 & 40 & 57 & 54 \\
\hline
\end{tabular}




\begin{tabular}{|c|c|c|c|c|c|c|c|}
\hline Preguntas & $\begin{array}{c}\text { Detractores } \\
0-6\end{array}$ & $\begin{array}{c}\text { Indiferentes } \\
7-8\end{array}$ & $\begin{array}{c}\text { Promotores } \\
9-10\end{array}$ & $\begin{array}{c}\% \\
\text { Detractores }\end{array}$ & $\begin{array}{c}\% \\
\text { Indiferentes }\end{array}$ & $\begin{array}{c}\% \\
\text { Promotores }\end{array}$ & $\begin{array}{l}\text { Índice De } \\
\text { Percepción }\end{array}$ \\
\hline $\begin{array}{l}\text { 17.- limpieza } \\
\text { establecimiento }\end{array}$ & 13 & 156 & 106 & 5 & 57 & 39 & 34 \\
\hline $\begin{array}{l}\text { 18.- instalaciones } \\
\text { establecimiento }\end{array}$ & 111 & 112 & 52 & 40 & 41 & 19 & -21 \\
\hline $\begin{array}{l}\text { 19.- estado } \\
\text { mobiliario }\end{array}$ & 93 & 128 & 54 & 34 & 47 & 20 & -14 \\
\hline 20.- señaléticas & 48 & 172 & 55 & 17 & 63 & 20 & 3 \\
\hline $\begin{array}{l}\text { 21.- satisfecho } \\
\text { servicios } \\
\text { recibidos }\end{array}$ & 20 & 121 & 134 & 7 & 44 & 49 & 42 \\
\hline
\end{tabular}

Fuente: Base de datos encuestas de satisfacción 2019

La encuesta de satisfacción al usuario está compuesta por 21 ítems de los cuales se toman 11 preguntas enfocadas en la satisfacción de usuarios las cuales están detalladas en la tabla 1.

En Consulta Externa se realizaron 275 encuestas a personas mayores de 18 años, en el primer trimestre del año 2019, los valores negativos reflejados en esta tabla son los que causan insatisfacción al paciente siendo la "Satisfacción del tiempo de espera para cita o turno" con valor negativo -20, lo cual señala que hay debilidades en el área de admisión y atención al usuario, al momento de brindar atención existen demoras en las citas médicas, situación que no solo depende del agendamiento de la unidad, ya que el $90 \%$, lo cubre un centro de llamadas, quedándose sin citas médicas grupos vulnerables, valor negativo que se toma como referencia, en razón de que en este factor se concentra la mayoría de las inconformidades. "Instalaciones del establecimiento" con -21, indica que el usuario no está conforme con la infraestructura del mismo, situación que se ha dado a conocer a las autoridades del Centro de Salud, encontrándose en un proceso de repotenciación para mejoras en lugar.

De -100 a 79\% se consideran como un indicador de no conformidad. Es aquí donde se implementan los ciclos de mejora continua que identifican los problemas, se involucra al personal, se establece tiempo de duración, propuesta de mejora, y un alcance cuantitativo y cualitativo.

A partir del $80 \%$ se toma en cuenta como un porcentaje aceptable.

Tabla 2

Índice de percepción área de emergencia

\begin{tabular}{|c|c|c|c|c|c|c|c|}
\hline Preguntas & $\begin{array}{c}\text { Detractores } \\
0-6\end{array}$ & $\begin{array}{c}\text { Indiferentes } \\
7-8\end{array}$ & $\begin{array}{c}\text { Promotores } \\
9-10\end{array}$ & $\begin{array}{c}\% \\
\text { Detractores }\end{array}$ & $\begin{array}{c}\% \\
\text { Indiferentes }\end{array}$ & $\begin{array}{c}\% \\
\text { Promotores }\end{array}$ & $\begin{array}{l}\text { Índice De } \\
\text { Percepción }\end{array}$ \\
\hline $\begin{array}{l}\text { 7.- satisfecho tiempo } \\
\text { espera establecimiento }\end{array}$ & 25 & 15 & 3 & 58 & 35 & 7 & -51 \\
\hline $\begin{array}{l}\text { 8.- facilidad llegar al } \\
\text { establecimiento }\end{array}$ & 23 & 14 & 6 & 53 & 33 & 14 & -39 \\
\hline $\begin{array}{l}\text { 12.- explicación estado de } \\
\text { salud }\end{array}$ & 1 & 28 & 14 & 2 & 65 & 33 & 31 \\
\hline 13.- trato & 1 & 30 & 12 & 2 & 70 & 28 & 26 \\
\hline $\begin{array}{l}\text { 16.- aseo presentación } \\
\text { personal funcionario } \\
\text { atención }\end{array}$ & 0 & 16 & 27 & 0 & 37 & 63 & 63 \\
\hline $\begin{array}{l}\text { 17.- limpieza } \\
\text { establecimiento }\end{array}$ & 2 & 32 & 9 & 5 & 74 & 21 & 16 \\
\hline
\end{tabular}




\begin{tabular}{l|ccccccc}
\multicolumn{1}{c}{ Preguntas } & $\begin{array}{c}\text { Detractores } \\
\mathbf{0 - 6}\end{array}$ & $\begin{array}{c}\text { Indiferentes } \\
\mathbf{7 - 8}\end{array}$ & $\begin{array}{c}\text { Promotores } \\
\mathbf{9 - 1 0}\end{array}$ & $\begin{array}{c}\text { \% } \\
\text { Detractores }\end{array}$ & $\begin{array}{c}\text { \% } \\
\text { Indiferentes }\end{array}$ & $\begin{array}{c}\text { \% } \\
\text { Promotores }\end{array}$ & $\begin{array}{c}\text { Índice De } \\
\text { Percepción }\end{array}$ \\
\hline $\begin{array}{l}\text { 18.- instalaciones } \\
\text { establecimiento }\end{array}$ & 24 & 19 & 0 & 56 & 44 & 0 & -56 \\
\hline 19.- estado mobiliario & 17 & 24 & 2 & 40 & 56 & 5 & -35 \\
\hline 20.- señaléticas & 10 & 32 & 1 & 23 & 74 & 2 & -21 \\
\hline $\begin{array}{l}\text { 21.- satisfecho servicios } \\
\text { recibidos }\end{array}$ & 2 & 35 & 6 & 5 & 81 & 14 & 9 \\
\hline
\end{tabular}

Fuente: Base de datos encuestas de satisfacción 2019

En Emergencia (Tabla 2), se realizaron 43 encuestas a personas mayores de 18 años, en el primer trimestre del año 2019, siendo los valores negativos reflejados en esta tabla los que causan insatisfacción al paciente, así: "Satisfacción del tiempo de espera en establecimiento" con un índice de percepción de -51, significa la falta de agilidad del servicio por parte de todos los profesionales de la unidad operativa, ya que representa un valor negativo y debe mejorarse mediante la implementación de estrategias o ciclos rápidos de mejora continua. "Facilidad para llegar al establecimiento" con -40, indica que el usuario tiene dificultades para llegar al establecimiento debido a que es un servicio que se brinda a la comunidad en general y acuden usuarios de diferentes partes de la ciudad, de otros cantones o comunas. "Instalaciones del establecimiento" con -56, indica que el usuario no está conforme con la infraestructura del mismo, situación que se dio a conocer a las autoridades del ente objeto de estudio, lo que conllevó a un proceso de repotenciación para mejoras en lugar, por ello se están brindando los servicios en otras instalaciones, lo que originó la suspensión del servicio de partos. "Estado de mobiliario" con -35, este servicio también debe ser mejorado según la percepción de los encuestados, por lo que se encuentra en proceso de cambio. "Señalética" con -21, resultado que evidencia la inconformidad de los usuarios al sistema de comunicación visual, ya que no les permite identificar y conocer la ubicación de cada área, servicio que debe ser mejorado considerando el ámbito cognitivo y la inclusión, herramienta que debe estar en varios lenguajes (señas, braile, español, inglés, entre otros).

Al igual que en consulta externa de -100 a $79 \%$ se considera como un indicador de no conformidad. Es aquí donde se implementan los ciclos de mejora continua, se identifican los problemas, se involucra al personal, se establece tiempo de duración, propuesta de mejora, y un alcance cuantitativo y cualitativo.

A partir del $80 \%$ se toma en cuenta como un porcentaje aceptable.

\subsection{Diagrama de Paretto}

Tabla 3

Encuestas de Satisfacción 2019 Diagrama de Paretto

\begin{tabular}{l|ccc}
\multicolumn{1}{c}{ Causa/problema/fenómeno } & Datos recolectados & Porcentaje & $\begin{array}{c}\text { Frecuencia } \\
\text { acumulada }\end{array}$ \\
\hline El tiempo de espera para ser atendido es extenso & 178 & 56 & 56 \\
\hline Incomodidad con la infraestructura del establecimiento & 87 & 27 & 83 \\
\hline Incomodidad con el estado del mobiliario & 32 & 10 & 53 \\
\hline Inadecuado direccionamiento, pocas señaléticas & 15 & 2 & 100 \\
\hline Difícil acceso para llegar a la casa de salud & 6 & 100 & 58 \\
\hline Total & 318 & & \\
\hline
\end{tabular}

Fuente: Base de datos encuestas de satisfacción 2019 
Figura 1

Encuestas de Satisfacción 2019 Diagrama de Pareto

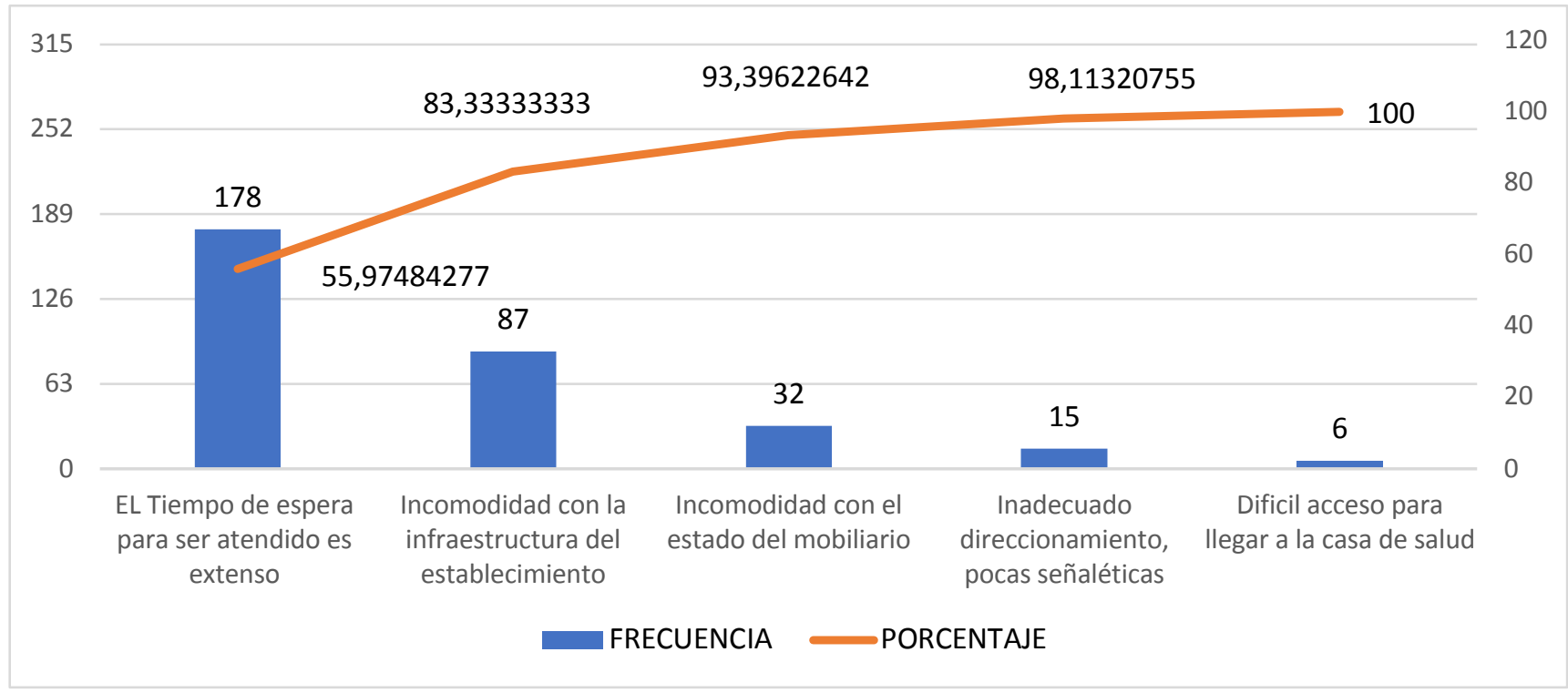

Fuente: Base de datos encuestas de satisfacción 2019

\subsection{Análisis}

Las 318 personas encuestadas afirman que el $80 \%$ de los problemas se concentran en el tiempo de espera para ser atendidos, y en la infraestructura del establecimiento de salud, por tal motivo se necesita un enfoque más profundo para resolver esta no conformidad que tienen los usuarios.

EL tiempo de espera para ser atendido es extenso.

Es el tiempo en el que el paciente previamente valorado, tiene que esperar para ser atendido por el médico, es decir ubicándole según el triaje mangester, en relación al área de emergencia.

Incomodidad con la infraestructura del establecimiento.

La infraestructura de la unidad de salud, resulta incómoda para el usuario y también para el personal que labora en la misma, para los primeros por el tiempo que les toca esperar para ser atendidos, esto está en relación directa con el ítem anterior.

\section{Conclusiones}

La Unidad Operativa Cuba Libre se ha caracterizado por brindar una amplia cartera de servicios a la comunidad, siendo la puerta de entrada el área de admisiones y estadística, el servicio al usuario empieza desde el personal de guardianía quienes tienen las funciones de guías.

Los usuarios llegan a las ventanillas en busca de atención de calidad y calidez, y en ocasiones no están conformes con lo recibido, entre ellos, demuestran inconformidad por el tiempo de espera, la infraestructura en la que se incluye el mobiliario no lo consideran cómodo, el sistema de señalética no les permite ubicarse o conocer la ruta a seguir, entre otros.

En resumen, pese a lo expuesto, los usuarios se sienten satisfechos por la gama de servicios que brinda la casa de salud a la comunidad, tanto en áreas de emergencia como consulta externa. 
De acuerdo al diagrama de Pareto se logró encontrar, que el $80 \%$ de problemas, en los cuales el usuario no está conforme, es el tiempo de espera que lo catalogó como excesivo.

\section{Referencias bibliográficas}

Arbeláez-Rodríguez, G., \& Mendoza, P. (2017). Relación entre gestión del director y satisfacción del usuario externo en centros de salud de un distrito del Ecuador. Paper presented at the Anales de la Facultad de Medicina.

Donabedian, A. (1993). Prioridades para el progreso en la evaluación y monitoreo de la calidad de la atención. Salud pública de México, 35(1), 94-97.

Fariño Cortez, J., Cercado Mancero, A., Vera Lorenti, E., Valle Flores, J., \& Ocaña Ocaña, A. (2018). Satisfacción de los usuarios y la calidad de atención que se brinda en las unidades operativas de atención primaria de salud. Revista Espacio, 39(32).

Guzmán, M. A., Ramos-Córdova, L. F., Castañeda-Sánchez, O., del Castillo-Sánchez, D. L., \& Gómez-Alcalá, A. V. (2006). Satisfaction of the Users at the Family Medicine Unit. Revista Médica del Instituto Mexicano del Seguro Social, 44(1), 39-45.

OMS. (2011). Guia para empleadores y representantes de los trabajadores.

Pública, M. S. (2019). Metodología para el cálculo del Índice de

Percepción de satisfacción del usuario/a externo., 6.

Seclén-Palacin, J., \& Darras, C. (2005). Satisfacción de usuarios de los servicios de salud: factores sociodemográficos y de accesibilidad asociados: Perú, 2000. Paper presented at the Anales de la Facultad de Medicina.

Varo, J. (1994). Gestión estratégica de la calidad en los servicios sanitarios: un modelo de gestión hospitalaria: Ediciones Díaz de santos.

Vivir, P. (2013). Plan nacional del buen Vivir. Recuperado el, 1(12), 2017.

Esta obra está bajo una Licencia Creative Commons Attribución-NoCommercial 4.0 International

(cc) BY-NC 